\title{
Intravenous chlorpromazine versus intramuscular sumatriptan for acute migraine
}

\author{
Anne-Maree Kelly, Michael Ardagh, Chris Curry, Jenny D’Antonio, Steven Zebic
}

\begin{abstract}
Objective-To establish whether there is any difference in the efficacy of a chlorpromazine regimen and a sumatriptan regimen for the management of the pain of acute severe migraine.

Setting-Two urban teaching hospital emergency departments.

Methods-Prospective, randomised, unblinded, crossover trial. All patients received intravenous metoclopramide $10 \mathrm{mg}$ and $1000 \mathrm{ml}$ of normal saline over $1 \mathrm{~h} ; 20$ were then randomised to receive intramuscular sumatriptan $6 \mathrm{mg}$ and 23 to receive intravenous chlorpromazine, 12.5 mg increments to a maximum of $37.5 \mathrm{mg}$. Response to treatment was measured using visual analogue pain scales.

Results-No difference in efficacy between the sumatriptan regimen and the chlorpromazine regimen was found. Adverse effects were mild and equally distributed between the groups.

Conclusions-The chlorpromazine and sumatriptan regimens studied are both very effective for the relief of the headache of severe migraine.

(f Accid Emerg Med 1997;14:209-211)
\end{abstract}

Keywords: migraine treatment; chlorpromazine; sumatriptan

Patients with severe migraine present to emergency departments infrequently but pose a considerable management dilemma. The pathophysiology of migraine is still not well understood and various treatments are used. These include narcotic analgesics, metoclopramide, dihydroergotamine, oral and parenteral non-steroidal anti-inflammatory agents, chlorpromazine, and sumatriptan. ${ }^{1}$ A review of published reports suggests that the two most effective agents (with respect to relief of pain and frequency of rebound headache) are intravenous chlorpromazine and intramuscular or subcutaneous sumatriptan. ${ }^{1}$

Current research suggests that the headache pain of migraine results from the activation of the trigeminovascular system. ${ }^{23}$ The triggers to the development of migraine headache are probably chemical and are thought to originate in the brain, blood vessel walls, and the blood itself. These triggers stimulate trigeminovascular axons, causing pain and the release of vasoactive neuropeptides from perivascular axons. In turn, these neuropeptides act on mast cells, endothelial cells, and platelets, resulting in increased extracellular concentrations of arachidonate metabolites, amines, peptides, and ions. These mediators and the resultant tissue injury lead to prolongation of pain and hyperalgesia. $^{2}$

Chlorpromazine is a phenothiazine used mainly for the treatment of psychiatric disorders. It is a powerful antagonist of the neurotransmitter action of dopamine in the basal ganglia and limbic system. It is also a potent antiemetic through its action on the chemoreceptor trigger zone. Its neuroleptic actions appear to alter pain perception. It is also an $\alpha$ adrenergic antagonist with some anticholinergic properties. The $\alpha$ blocking action of chlorpromazine can result in orthostatic hypotension. Chlorpromazine acts as an antagonist at both histamine and 5-hydroxytryptamine (5-HT) receptors. ${ }^{4}$ During short term use, the side effects of chlorpromazine are dose dependent orthostatic hypotension, lowering of the seizure threshold, tremors, and drowsiness. Dystonia is an idiosyncratic reaction and may occur after a single dose. ${ }^{4}$ The mechanism of action of chlorpromazine in migraine is uncertain. It is possibly the result of a combination of actions, including the anti5-HT effect, an antidopamine effect in the chemoreceptor trigger zone, and vascular effects through its $\alpha$ blocking action. ${ }^{5}$ The reported success rate of chlorpromazine regimens in the treatment of migraine varies from $47 \%$ to $96 \% .^{5-9}$

Sumatriptan is a specific and selective 5-HT (subtype 1D) agonist that has no effect on other 5-HT receptor subtypes. This receptor is found predominantly in cranial blood vessels and when stimulated produces constriction of large blood vessels which may be dilated during attacks of migraine.$^{10}$ Clinical response begins within 10-15 minutes of subcutaneous injection. ${ }^{11}$ Adverse effects include drowsiness, weakness, dizziness, flushing, rash, pruritus, increase in blood pressure, chest pain, and chest tightness. Sumatriptan is contraindicated in patients with a history of ischaemic heart disease and uncontrolled hypertension and in those using ergot preparations. ${ }^{11}$ The antimigraine effect of sumatriptan is thought to be due to its effect on the 5-HT subtype 1D receptors in cranial blood vessels. ${ }^{1012}$ The reported success rate of sumatriptan regimens varies from $49 \%$ to $80 \% .^{13-15}$

There is a considerable cost difference between these agents, with an ampoule of chlorpromazine costing Australian hospital pharmacies $\mathrm{A} \$ 0.71$, compared with $\mathrm{A} \$ 47.31$ for an ampoule of sumatriptan. 
As these agents have not previously been compared in a prospective clinical trial, this study was designed to establish whether there is any difference in the efficacy of a chlorpromazine regimen and a sumatriptan regimen for managing the pain of acute severe migraine.

\section{Methods}

This study was conducted as a randomised, prospective, unblinded crossover trial. Subjects were enrolled from the emergency departments of Western Hospital Footscray, Melbourne, Australia and Christchurch Hospital, Christchurch, New Zealand. Approval of the ethics committees of both institutions was obtained.

The inclusion criteria were: age $18-65$ years, features typical of migraine, past history of migraine headache, and no impairment of conscious state. Exclusion criteria were: headache not typical of migraine, allergy to any of the study agents, inability to mark a visual analogue pain scale, the presence of abnormal neurological signs, failure to give informed consent, and impairment of conscious state.

All patients, after giving informed consent, were asked to indicate the level of their pain on a $10 \mathrm{~cm}$ non-hatched visual analogue pain scale, marked from " 0 " at one end to " 10 " at the other. Patients were verbally instructed that " 0 " meant "no pain" and " 10 " meant "worst pain ever".

Each then received metoclopromide $10 \mathrm{mg}$ intravenously and an infusion of normal saline was started, $1000 \mathrm{ml}$ to be given over one hour. The normal saline infusion was used to counter the dehydration which is commonly associated with severe migraine. Metoclopromide was given for its anti-nausea effects. The researchers were aware that metoclopramide might of itself have an impact on migraine, with reported success rates for its use of $0-60 \%$ (mean value approximately $40 \%) .^{16-19}$ As this was consistently much lower than the reported success rates of either sumatriptan or chlorpromazine in previous studies, we chose not to include a separate metoclopramide-alone arm for ethical reasons.

Patients were then randomised by date of presentation to receive either chlorpromazine $12.5 \mathrm{mg}$ intravenously, repeated at 25 minutes and 45 minutes if needed, or sumatriptan $6 \mathrm{mg}$ intramuscularly. This was given within five minutes of the metoclopromide. On odd dates patients received the sumatriptan regimen and on even dates the chlorpromazine regimen. The study design determined that if, two hours after administration of the trial drug, pain was still rated at more than $30 \%$ of the length of the visual analogue scale, the patient was to be crossed over to the other agent.

Pain was measured using the visual analogue scale at enrolment and one and two hours after administration of the trial drug. The study definition of treatment success was relief of pain to the patient's satisfaction. If pain was controlled to the patient's satisfaction two hours after last medication, they were discharged home. If not, they were withdrawn from the study for further investigation and
Table 1 Demographic characteristics of trial groups

\begin{tabular}{llll}
\hline Group & Average age & Female: male & Total \\
\hline Sumatriptan & 32 & $12: 8$ & 20 \\
Chlorpromazine & 35 & $17: 6$ & 23 \\
\hline
\end{tabular}

management. The occurrence of adverse events was recorded.

\section{Results}

Forty three patients were enrolled in the study, 20 in the sumatriptan group and 23 in the chlorpromazine group. The age and gender distribution in the groups is shown in table 1.

Mean pain scores at enrolment and at one hour and two hours after treatment are shown in table 2 . The data were analysed using analysis of variance. The hypothesis that there was no difference in efficacy between the regimens was accepted at the 0.05 level of significance.

The close similarity in efficacy is also shown by the proportion of patients in each group who were pain-free (that is, VAS score $=0$ ) at each time interval. Ten per cent of the patients in the sumatriptan group were pain-free at one hour compared to $18 \%$ of the chlorpromazine group. Forty two per cent of the sumatriptan group were pain-free at two hours, compared to $41 \%$ of the chlorpromazine group. Relief of pain to the patient's satisfaction with the sumatriptan regimen occurred in 19/20 (95\%) and with the chlorpromazine regimen in $22 / 23$ (95\%). Only one patient from each group failed to achieve analgesia to their satisfaction. One of these was crossed over to the other arm of the study and following treatment with the alternative drug continued to report moderate headache. The other patient declined further treatment.

Six occurrences of adverse events were reported: three in the sumatriptan group and three in the chlorpromazine group. All were mild, did not require specific treatment, and resolved within the study period. In the sumatriptan group the adverse symptoms reported were nausea (1), burning in the face (1), and unpleasant dreams (1). In the chlorpromazine group the events were dizziness (1), fever (1), and one transient episode of palpitations (sinus tachycardia). The patient who experienced transient fever had a normal CSF examination and white cell count. No infective focus was identified. Of particular note, no episode of a dystonic reaction occurred in either group.

\section{Discussion}

Migraine headache can be a disabling condition. Most migraine headaches are successfully managed by the patient and their general practitioner; however, a few fail to respond and present to emergency departments. As most patients have tried oral medications before attending, parenterally administered agents are most appropriate for use in emergency departments. A recent review of published reports suggests that the two most effective agents (with respect to relief of pain and incidence of 
Table 2 Mean pain scores with 95\% confidence intervals for each regime

\begin{tabular}{llll}
\hline & \multicolumn{4}{l}{ Group mean pain scores (95\% CI) } \\
\cline { 2 - 4 } & At presentation & At 1 hour & At 2 hours \\
\hline Sumatriptan & $74.6(67.3$ to 81.9$)$ & $25.0(16.3$ to 33.7$)$ & $11.3(3.6$ to 19.0$)$ \\
Chlorpromazine & $75.7(68.8$ to 82.6$)$ & $27.9(16.3$ to 39.4$)$ & $21.4(9.4$ to 33.4$)$ \\
\hline
\end{tabular}

CI, confidence interval.

rebound headache) are intravenous chlorpromazine and parenteral sumatriptan.

In this study, there was no difference in mean score, the proportion of patients who were pain-free at each interval, and the rate of adverse events between the group which received the sumatriptan regimen and that which received the chlorpromazine regimen. These success rates of the regimens are consistent with those reported in other studies of chlorpromazine and sumatriptan.

It might be suggested that the metoclopramide given as part of each study regimen was responsible for the observed analgesic effect. This is unlikely as previous studies investigating the efficacy of metoclopramide in migraine have reported effective analgesia rates of between 0 and $67 \%$ (with a mean of $\sim 40 \%$ ). ${ }^{16-19}$ This is much lower than the $95 \%$ effective analgesia rates of both of the two regimens studied here. The most recent study investigating the efficacy of metoclopramide in migraine $^{19}$ reported that $69 \%$ of patients had "more than $70 \%$ relief of pain" and that $25 \%$ of patients reported complete relief of headache at 45 minutes. The doses of metoclopramide used were, however, much larger than in our study, averaging approximately $16 \mathrm{mg}$. Despite this larger dose, the treatment success rates of the regimens in our study are higher $(P$ $<0.05$ ).

The fact that chlorpromazine is effective in the treatment of migraine despite its anti-5-HT activity is particularly interesting. It suggests that there may be more than one way to interrupt the complex mechanisms responsible for the genesis of migraine.

Chlorpromazine is much cheaper than sumatriptan (in Australia, $1.5 \%$ of the cost); however, because of the possibility of orthostatic hypotension an intravenous line and fluid are usually used with it. Even allowing for this, the chlorpromazine regimen remains the cheaper option. Sumatriptan is given as an intramuscular injection without the need for an intravenous line and can be self administered if thought appropriate. Chlorpromazine, on the other hand, requires intravenous access, with the consequent infection risk and the need for more close observation. Many patients have been suffering their migraine for some hours before presentation and have also been vomiting, resulting in a degree of dehydration. The chlorpromazine regimen does include some fluid replacement which may be of independent benefit. Given the similarity in efficacy of these agents, the choice of agent will therefore depend on considerations of cost, convenience, and the management of dehydration.

\section{CONCLUSION}

Both the sumatriptan and chlorpromazine regimens studied are highly effective for the treatment of the pain of migraine. The chlorpromazine regimen studied is as effective as the sumatriptan regimen for the relief of the headache of severe migraine and at a lower cost.

1 Kelly AM, Bryant MG, Zebic S. The emergency department treatment of severe migraine Emerg Med $1995 ; 7: 162-9$

2 Moskowitz MA. Neurogenic inflammation in the pathophysiology and treatment of migraine. Neurology 1993; 43(suppl 3):16-20.

3 Goadsby PJ, Edvinsson L. The trigeminovascular system and migraine: studies characterising cerebrovascular and neuropeptide changes seen in humans and cats. Ann Neurol 1993;33:48-56.

4 Gilman AG, Rall TW, Nies AS, Taylor P. The pharmacological basis of therapeutics, 8th ed. London: Pergamon Press, cal basis

5 Lane PL, McLellan BA, Baggoley CJ. Comparative efficacy of chlorpromazine and meperidine with dimenhydramine in migraine headache. Ann Emerg Med 1989;18:360-5.

6 Lane PL, Ross R. IV chlorpromazine: preliminary results in acute migraine. Headache 1985;25:302-4.

7 Iserson KV. Parenteral chlorpromazine treatment of migraine. Ann Emerg Med 1983;12:756-8.

8 McEwen JI, O'Connor HM, Dinsdale HB. Treatment of migraine with intramuscular chlorpromazine. Ann Emerg Med 1987;16:758-63.

9 Bell R, Montoya D, Shuaib A, Lee MA. A comparative trial of three agents in the treatment of acute migraine headache. Ann Emerg Med 1990;19:1079-82.

10 Abramowicz $M$, ed. Sumatriptan for migraine. Med Lett 1992;34:91-3.

11 Australian prescription products guide, 25th ed. Victoria: Australian Pharmaceutical Publishing Company, 1995.

12 Buzzi MG, Moskowitz MA. Evidence for 5-HT-1B/1D receptors mediating the antimigraine effect of sumatriptan and dihydroergotamine. Cephalgia 1991;11:165-8.

13 Cady RK, Wendt JK, Kirchner JR, Sargeant JD, Rothrock JF, Skaggs H. Treatment of acute migraine with subcutaneous sumatriptan. JAMA 1991;265:2831-5.

14 The Subcutaneous Sumatriptan International Study Group. Treatment of migraine attacks with sumatriptan. N Engl J Med 1991;325:316-21.

15 Akpunonu BE, Mutgi AB, Federman DJ, Volinsky FG, Brickman K, Davis RL, et al. Subcutaneous sumatriptan for the treatment of acute migraine in patients admitted to the emergency department: a multicenter study. Ann Emerg Med 1995;25:464-9.

16 Tek DS, McClellan DS, Olshaker JS, Allen CL, Arthur DC. A prospective, double blind study of metoclopramide hydrochloride for the control of migraine in the emergency hydrochloride for the control of migraine in the

17 Tfelt-Hansen P, Olesen J, Aebelholt-Krabbe A, Melgaard B, Veillis B. A double blind study of metoclopramide in migraine attacks. J Neurol Neurosurg Psychiatr 1980:43: 369-71.

18 Jones JE, Chun E, Pack S. Intramuscular prochlorperazine versus metoclopramide for the treatment of acute migraine headache [abstr]. Ann Emerg Med 1995;25:154

19 Cameron JD, Lane PL, Speechley M. Intravenous chlorpromazine versus intravenous metoclopramide in acute migraine headache. Ann Emerg Med 1995;2:597-602. 